\title{
Correction to: Resilience Strategies and the Pharmaceutical Supply Chain: The Role of Agility in Mitigating Drug Shortages
}

Emilia Vann Yaroson, Liz Breen, Jiachen Hou and Julie Sowter

\section{Correction to:}

Chapter "Resilience Strategies and the Pharmaceutical Supply Chain: The Role of Agility in Mitigating Drug Shortages" in: A. P. Barbosa-Povoa et al. (eds.), Pharmaceutical Supply Chains-Medicines Shortages, Lecture Notes in Logistics, https://doi.org/10.1007/978-3-030-15398-4_18

In the original version of this book, the following belated correction has been incorporated: Co-author names have been included in Chapter "Resilience Strategies and the Pharmaceutical Supply Chain: The Role of Agility in Mitigating Drug Shortages". The corrected chapter has been updated with the changes. 